\title{
SOP 06: Selecting the Participating Centers and Activation of a Trial
}

\section{Background}

If there are more candidates than the planned maximum number of participating study centers, a selection will be made as outlined in this SOP.

The Chairperson(s) of the respective working group will announce the participating centers in consultation with the sponsor after he/she has received responses from all centers to whom participation was offered.

\section{Selection of Suitable Trial Centers}

ICH-GCP defines the conditions that must be met by investigators and study centers participating in clinical trials that comply with Good Clinical Practice (GCP). According to these guidelines, it is the responsibility of the sponsor to select suitable centers and to continuously monitor their suitability throughout the study. This responsibility is shared by the monitor as the principal communication link between investigators and the sponsor. Responsibilities of the investigator besides his/her professional qualifications and a thorough familiarity with the properties of the investigational product are described in section 4.1.2 of ICHGCP. The criteria to be met by trial centers are summarized in the Selection Criteria for Membership in a clinical Working Group of CESAR (see 'SOP 01: Clinical Investigations in CESAR').

Each study center participating in a multicenter trial must satisfy the above criteria. A uniform level of information of all participants must be ensured by establishing proper procedures for data flow among all parties involved (see 'SOP 7: Data Flow, Monitoring, and Archiving').

The Chairperson(s) of the respective working group, in consultation with the sponsor, selects the centers to participate in a study that is to be conducted under the auspices of CESAR. This holds in particular if more centers wish to participate than is reasonable for a given study.
In order to comply with the principles of GCP, it is necessary to carefully coordinate activities both before starting a trial and throughout its duration. For practical reasons, there will be a limit for the maximum number of centers that can participate in a trial, to ensure adequate monitoring and to minimize differences among study centers, e.g. regarding patient selection and assessments. For reasons to exclude a center from an ongoing study; see below.

\section{Competing Studies}

If CESAR has several disease-oriented studies with overlapping patient selection criteria ongoing at the same time, each center may participate in only one of them. This holds for phase II/III trials only.

In order to minimize a selection bias and the duration of a study, it is expected that an institution will not participate in a study of CESAR if it is concurrently engaged in a non-CESAR study with similar selection criteria.

\section{Exclusion from an Ongoing Study}

The Coordinating Investigator (CI) can exclude a participating center from further patient accrual for an ongoing trial in case of the following deficiencies:

- Significant failure to achieve the predicted rate of patient accrual.

- Repeated nonadherence to the trial protocol despite documented reminders.

- Repeated delays in the completion and submission of Case Report Forms.

- Submission of data that cannot be validated.

- Repeated accrual of ineligible patients.

- Repeated failure of the (principal) investigator or his authorized deputy to be available during site visits by the monitor or the extramural review board. 
- Repeated failure to cooperate in quality assurance measures as defined in 'SOP 11. Quality Assurance'.

\section{Study Activation}

A study will be activated once the following criteria have been satisfied:

- The CI has integrated the change requests from the Project Committee and participating working group members into a final version of the protocol.
- A sufficient number of working group members have declared their interest to participate in the clinical trial.

- Approval by the Ethics Committee that is relevant for the CI.

- Written information of all participating centers of the study.

- The sponsor has met all applicable regulatory prerequisites (insurance, notification of the trial to regulatory authorities, drug import certification, etc.).

Patient accrual at a center can start after the initiation visit by the monitor. 